\title{
Autoclinisms and automorphisms of finite groups
}

\section{Ratje Reimers and Jürgen Tappe}

Let $\Gamma$ be a family of isoclinic finite groups, and let acl( $\Gamma)$ be the group of autoclinisms of $\Gamma$. In this paper we prove the following formula:

$$
\frac{1}{|\operatorname{acl}(\Gamma)|}=\sum \frac{1}{|\operatorname{aut}(\Gamma)|}
$$

where the sum is taken over a complete system of stem groups $S$ in $\Gamma$. This result is due to $P$. Hall, who outlined a proof in his paper, "On groups of automorphisms" ( $J$. reine angew. Math. 182 (1940), 194-204), using presentations, whereas in this paper we consider stem groups in terms of central extensions.

\section{Isomorphisms of central extensions}

Let $K$ be a finite group and $A$ a finite abelian group. Let $r: K \times K \rightarrow A$ be a factor set which belongs to the trivial operation of $K$ on $A$ (cf. Huppert [4], p. 87). Then

$$
(A, K, r)=\{(a, k) \mid a \in A, k \in K\}
$$

with the multiplication

$$
(a, k)(b, l)=(a b r(k, l), k l)
$$

is a group. $\{(a, 1) \mid a \in A\}$ is a subgroup of the centre of $(A, K, r)$, and we obtain

$$
\{(a, 1) \mid a \in A\} \simeq A \text { and }(A, K, r) /\{(a, 1) \mid a \in A\} \simeq K .
$$

Let $R=(A, K, r)$ and $S=(A, K, s)$. In order to distinguish the

Receired 10 September 1975. The authors wish to express their thanks to Professor J. Neubüser for his kind and helpful advice.

439 
elements of $R$ and $S$, we indicate them and write $(a, k)_{R}$ for the elements of $R$ and $(a, k)_{S}$ for the elements of $S$. Let

$$
A_{R}=\left\{(a, 1)_{R} \mid a \in A\right\} \text { and } A_{S}=\left\{(a, 1)_{S} \mid a \in A\right\} \text {, }
$$

and let $\lambda$ be an isomorphism from $R$ to $S$, such that

$$
A_{R}^{\lambda}=A_{S}
$$

Then $\lambda$ induces an automorphism $\alpha$ of $K$ and an automorphism $\beta$ of $A$ by

$$
A_{S}(1, k)_{R}^{\lambda}=A_{S}\left(1, k^{\alpha}\right)_{S}
$$

and

$$
(\alpha, 1)_{R}^{\lambda}=\left(a^{\beta}, 1\right)_{S}
$$

Let $t: K \times K \rightarrow A$ be a factor set, and let $(\alpha, \beta)$ be an element of $\operatorname{aut}(K) \times \operatorname{aut}(A)$. Then we define

$$
t^{(\alpha, \beta)}:=\left((k, l) \mapsto\left(t\left[k^{\alpha^{-1}}, z^{\alpha^{-1}}\right)\right)^{\beta}\right) \text {. }
$$

It is easy to see that $t^{(\alpha, \beta)}$ is also a factor set, and we have the following result:

LEMMA 1.3. Let $R=(A, K, r)$ and $S=(A, K, s)$.

(i) Let $\lambda$ be an isomorphism from $R$ to $S$ which satisfies (1.1) and which induces $\alpha \in$ aut $(K)$ and $\beta \in$ aut $(A)$. Then the factor sets ${ }_{r}(\alpha, \beta)$ and $s$ are equivalent.

(ii) Let $(\alpha, \beta) \in \operatorname{aut}(K) \times \operatorname{aut}(A)$, and assume that $r^{(\alpha, \beta)}$ and $s$ are equivalent. Then there exists an isomorphism from $R$ to $S$ which satisfies (1.I) and induces $\alpha$ and $B$.

Proof. ( $i)$ As $\lambda$ satisfies (1.1), there is a function $c: K \rightarrow A$, such that we have for all $a \in A$ and $k \in K$,

$$
(a, k)_{R}^{\lambda}=\left(a^{\beta} c(k), k^{\alpha}\right)_{S} \text {. }
$$

As $\lambda$ is an isomorphism, we have for all $k, \imath \in K$, 


$$
\begin{aligned}
& \left((1, k)_{R}(1, l)_{R}\right)^{\lambda}=(1, k)_{R}^{\lambda}(1, l)_{R}^{\lambda}, \\
& (r(k, l), k l)_{R}^{\lambda}=\left(c(k), k^{\alpha}\right)_{S}\left(c(l), l^{\alpha}\right)_{S},
\end{aligned}
$$

and

$$
\left((r(k, z))^{\beta} c(k \tau), k^{\alpha} z^{\alpha}\right)_{S}=\left(c(k) c(\tau) s\left(k^{\alpha}, z^{\alpha}\right), k^{\alpha} z^{\alpha}\right)_{S} .
$$

This implies

$$
(r(k, \tau))^{\beta} c(k \tau)=s\left(k^{\alpha}, z^{\alpha}\right) c(k) c(l),
$$

and $(i)$ follows immediately.

(ii) Assume that there is a function $d: K \rightarrow A$ such that

$$
\left(r\left(k^{\alpha^{-1}}, z^{\alpha^{-1}}\right)\right)^{\beta} d(k z)=s(k, z) d(k) d(z)
$$

holds for all $k, \imath \in K$. Hence, we have

$$
(r(k, z))^{\beta} d\left(k^{\alpha} z^{\alpha}\right)=s\left(k^{\alpha}, z^{\alpha}\right) d\left(k^{\alpha}\right) d\left(z^{\alpha}\right) .
$$

The calculations in the proof of (i) show that the mapping $\lambda: R \rightarrow S$ defined by

$$
(a, k)_{R}^{\lambda}=\left(a^{\beta} d\left(k^{\alpha}\right), k^{\alpha}\right)_{S}
$$

is an isomorphism which satisfies (1.1).

\section{Central automorphisms and symmetric factor sets}

Let $G$ be a finite group with the centre $Z(G)$ and the commutator subgroup $G^{\prime}$. An automorphism of $G$ is called a central automorphism, if it induces the identity on $G / Z(G)$, and let $C(G)$ denote the group of all central automorphisms of $G$.

A finite group $G$ is called a stem group, if $Z(G)$ is contained in $G^{\prime}$. As stem groups have no direct abelian factor, we obtain from Sanders [6],

$$
C(G) \simeq \operatorname{hom}\left(G / G^{\prime}, Z(G)\right),
$$

where $\operatorname{hom}\left(G / G^{\prime}, Z(G)\right)$ denotes the group of homomorphisms from $G / G^{\prime}$ to $Z(G)$. 
Let $K$ be a finite group and $A$ a finite abelian group. Let

$$
\operatorname{Sym}(K, A)
$$

denote the set of all factor sets $f: K \times K \rightarrow A$ which belong to the trivial operation of $K$ on $A$ and which satisfy

$$
f(x, y)=f(y, x) \text { for all } x, y \in K,
$$

and let

$$
\operatorname{sym}(K, A)
$$

denote the set of all $f \in \operatorname{Sym}(K, A)$, which satisfy

$$
f(k, x)=f(x, k)=1 \text { for all } k \in K^{\prime} \text {. }
$$

Let $\overline{\operatorname{sym}}(K, A)$ and $\overline{\operatorname{sym}}(K, A)$ be the corresponding subgroups of the second cohomology group $H^{2}(K, A)$. It is easy to see that

$$
\overline{\operatorname{sym}}(K, A) \simeq \overline{\operatorname{sym}}\left(K / K^{\prime}, A\right) \text {. }
$$

From Fuchs [1], pp. 238, 243, and 248, and Reimers [5], p. 30, we obtain

$$
\overline{\operatorname{Sym}}\left(K / K^{\prime}, A\right) \simeq \operatorname{hom}\left(K / K^{\prime}, A\right) \text {. }
$$

These relations and (2.1) yield for all stem groups $G$,

$$
C(G) \simeq \overline{\operatorname{sym}}(G / Z(G), Z(G)) \text {. }
$$

\section{Isoclinisms of stem groups}

DEFINITION 3.1 (Hall). Let $G$ and $H$ be finite groups. $G$ and $H$ are called isoclinic, if and only if there exist isomorphisms $\sigma$ and $\tau$,

$$
\begin{aligned}
& \sigma: G / Z(G) \rightarrow H / Z(H), \\
& \tau: G^{\prime} \rightarrow H^{\prime},
\end{aligned}
$$

such that

$$
\left[g_{1}, g_{2}\right]^{\tau}=\left[h_{1}, h_{2}\right], h_{1} \in\left(z(G) g_{1}\right)^{\sigma}, h_{2} \in\left(z(G) g_{2}\right)^{\sigma}
$$

for $g_{1}, g_{2} \in G$. The pair $(\sigma, \tau)$ is called an isoclinism from $G$ to $H$. The classes of isoclinic groups are called families.

Hall proved in [2] that each family $\Gamma$ contains stem groups, and that a group in $\Gamma$ is a stem group if and only if it has minimal order. One can also show that the centres of isoclinic stem groups are isomorphic. 
Hence, we have a group $K$ and an abelian group $Z$ such that each stem group in $\Gamma$ is isomorphic to a group of the following form:

$$
R=(Z, K, r) \text {, where } Z(R)=Z_{R} \text { and } R^{\prime}=\left(z, K^{\prime}, r^{\prime}\right) \text {, }
$$

and where $r^{\prime}$ is tue restriction of $r$ to $K^{\prime} \times K^{\prime}$.

Let $S=(Z, K, s)$, and let $(\sigma, \tau)$ be an isoclinism from $R$ to $S$. Then we have $z_{R}^{\tau}=z_{S}$ and $z_{S}=Z(S)$, and $(\sigma, \tau)$ induces automorphisms $\alpha \in \operatorname{aut}(K)$ and $\beta \in \operatorname{aut}(Z)$ by

$$
\left(z_{R}(1, k)_{R}\right)^{\sigma}=z_{S}\left(1, k^{\alpha}\right)_{S},
$$

and

$$
(z, 1)_{R}^{\tau}=\left(z^{\beta}, 1\right)_{S}
$$

THEOREM 3.2. Let $R=(z, K, r)$ and $S=(z, K, s)$, and let $Z(R)=Z_{R} \subseteq R^{\prime}$.

(i) Assume that there exists an isoclinism from $R$ to $S$ which induces $(\alpha, \beta) \in \operatorname{aut}(K) \times \operatorname{aut}(2)$. Then there exists a factor set $f \in \operatorname{sym}(K, Z)$ such that $r^{(\alpha, \beta)}$ and sf are equivalent.

(ii) Let $(\alpha, \beta) \in \operatorname{aut}(K) \times \operatorname{aut}(z)$ and $f \in \operatorname{sym}(K, z)$ such that ${ }_{r}(\alpha, \beta)$ and sf are equivalent. Then there exists an isoclinism from $R$ to $S$ which induces $\alpha$ and $B$.

Proof. (i) Let $(\sigma, \tau)$ be the isoclinism from $R$ to $S$ which induces $(\alpha, \beta)$. By the definition of isoclinism $\tau$ induces $\alpha$ on $K^{\prime}$, and we have a function $c: K^{\prime} \rightarrow Z$ such that

$$
\left(z, k^{\prime}\right)_{R}^{\tau}=\left(z^{\beta} c\left(k^{\prime}\right), k^{\prime \alpha}\right)_{S}
$$

holas for all $k^{\prime} \in K^{\prime}, z \in Z$. As in the proof of Lemma 1.3 we have for a.ll $k^{\prime}, l^{\prime} \in K^{\prime}$ :

$$
\left(r\left(k^{\prime}, z^{\prime}\right)\right)^{\beta} c\left(k^{\prime} z^{\prime}\right)=s\left(k^{\prime \alpha}, z^{\prime \alpha}\right) c\left(k^{\prime}\right) c\left(z^{\prime}\right) .
$$

Let $K=\mathrm{U} K^{\prime} k_{i}$, where $k_{1}=1$, let $k=k^{\prime} k_{i}, k^{\prime} \in K^{\prime}$, and set 


$$
b(k)=r^{(\alpha, \beta)}\left(k^{\prime}, k_{i}\right) s^{-1}\left(k^{\prime}, k_{i}\right)\left(c\left(k^{\prime \alpha^{-1}}\right)\right)^{-1} .
$$

For all $k^{\prime} \in K^{\prime}$ and all $k_{i}$ we have

$$
b\left(k^{\prime}\right)=\left(c\left(k^{\prime \alpha^{-1}}\right)\right)^{-1} \text { and } b\left(k_{i}\right)=1 \text {. }
$$

Now we consider the following factor set $f$, where

$$
f(k, \tau)=r^{(\alpha, \beta)}(k, l) s^{-1}(k, z) b(k) b(\tau)(b(k l))^{-1} .
$$

By $(3.3),(3.4)$, and $(3.5)$ we obtain for all $k_{i}$ and all $k^{\prime}, l^{\prime} \in K^{\prime}$,

$$
f\left(k^{\prime}, k_{i}\right)=1 \text { and } f\left(k^{\prime}, z^{\prime}\right)=1 \text {. }
$$

This yielis, together with the folloring relation (see Huppert [4], p. 87)

$$
f\left(k^{\prime}, Z^{\prime} k_{i}\right)=f\left(k^{\prime} Z^{\prime}, k_{i}\right) f\left(k^{\prime}, z^{\prime}\right)\left(f\left(z^{\prime}, k_{i}\right)\right)^{-1},
$$

that

$$
f\left(k^{\prime}, \tau\right)=1 \text { for ali } k^{\prime} \in K^{\prime} \text { and } l \in K \text {. }
$$

Let $k, l \in K$ and

$$
\left[\left(1, k^{\alpha^{-l}}\right)_{R},\left(1, z^{\alpha \cdot 1}\right)_{R}\right]=\left(x,[k, z]^{\alpha^{-1}}\right)_{R}
$$

As $R$ and $S$ are isocinic, we have

$$
\left[(1, k)_{S},(1, z)_{S}\right]=\left(x,[k, z]^{\alpha^{-1}}\right)_{R}^{\tau}=\left(x^{\beta} c\left([k, z]^{\alpha^{-1}}\right),[k, z]\right)_{S} .
$$

Hence, we obtain in $T=(2, K, t)$, where $t=r^{(\alpha, \beta)} s^{-1}$,

$$
\left[(1, k)_{T},(1, z)_{T}\right]=\left(\left(c\left([k, z]^{\alpha^{-1}}\right)\right)^{-1},[k, l]\right)_{T}
$$

We have $f(k, Z)=t(k, Z) \hat{b}(k) b(\tau)(z(k \zeta))^{-1}$, and by some calculations we obtain in $F=(z, K, f)$,

$$
\left[(1, k)_{F},(1, z)_{F}\right]=\left(\left\{c\left([k, z]^{\alpha-1}\right)\right)^{-1}(b([k, l]))^{-1},[k, l]\right)_{F},
$$

and (3.5) yields 


$$
\left[(1, k)_{F},(1, l)_{F}\right]=(1,[k, l])_{F}
$$

We have

$$
\begin{aligned}
& (f(k, l), k l)_{F}=(1, k)_{F}(1, l)_{F}, \\
(f(k, l), k l)_{F}= & {\left[(1, k)_{F}^{-1},(1, l)_{F}^{-1}\right](1, l)_{F}(1, k)_{F} } \\
= & {\left[(1, k)_{F}^{-1},(1, \tau)_{F}^{-1}\right](f(l, k), l k)_{F} } \\
= & \left(1,\left[k^{-1}, l^{-1}\right]\right)_{F}(f(l, k), l k)_{F} \text { by }(3.8) \\
= & \left(f(l, k) f\left(\left[k^{-1}, l^{-1}\right], \tau k\right),\left[k^{-1}, l^{-1}\right] \tau k\right)_{F} \\
= & (f(l, k), k l)_{F} \text { by }(3: 7) .
\end{aligned}
$$

Hence, we obtain for all $k, l \in K$,

$$
f(k, z)=f(z, k) \text {. }
$$

By (3.7) and (3.9) we have $f \in \operatorname{sym}(K, Z)$, and (3.6) yields that $r^{(\alpha, \beta)}$ and $s f$ are equivalent.

(ii) Let us define $\sigma: R / Z_{R} \rightarrow S / Z_{S}$ by

$$
\left(z_{R}(1, k)\right)^{\sigma}=z_{S}\left(1, k^{\alpha}\right)
$$

Let $a: K \rightarrow Z$ oe such that

$$
r^{(\alpha, \beta)}(k, Z) a(k Z)=s(k, Z) f(k, Z) a(k) a(Z) .
$$

As $f\left(k^{\prime}, l^{\prime}\right)=I$ for all $k^{\prime}, l^{\prime} \in K^{\prime}$, we obtain as in the proof of Lemma 1.3 that $\tau: R^{\prime} \rightarrow S$, where

$$
\left(z, k^{\prime}\right)^{\tau}=\left(z^{\beta} a\left(k^{\prime \alpha}\right), k^{\prime \alpha}\right) \text { for all } z \in Z, k^{\prime} \in K^{\prime},
$$

is an injective homomorphism.

The factor set $f$ satisfies (3.7) and (3.9). Hence, the proof of (3.9) shows that (3.8) is valid, too. Let $v=s f$ and $V=(Z, K, v)$. From (3.8) we obtain that

$$
\left[(1, k)_{S},(1,2)_{S}\right]=(x,[k, 2])_{S}
$$

implies 


$$
\left[(1, k)_{V},(1, l)_{V}\right]=(x,[k, l])_{V} .
$$

One can show by (3.10) that

$$
\left[(1, k)_{R},(1, l)_{R}\right]=(y,[k, l])_{R}
$$

implies

$$
\left[\left(1, k^{\alpha}\right)_{V},\left(1, z^{\alpha}\right)_{V}\right]=\left(y^{\beta} \alpha\left([k, z]^{\alpha}\right),[k, z]^{\alpha}\right)_{V}
$$

Hence, we have

$$
\left[(1, k)_{R},(1, l)_{R}\right]^{\tau}=\left[\left(1, k^{\alpha}\right)_{S},\left(1, l^{\alpha}\right)_{S}\right]
$$

We also obtain

$$
R^{\prime \tau}=S^{\prime},
$$

and in order to prove that $(\sigma, \tau)$ is the desired isoclinism, it remains to show

$$
z_{S}=z(S)
$$

Let $(z, k)_{S} \in Z(S)$. Then we have

$$
\left[(z, k)_{S},(1, l)_{S}\right]=\left[(1, k)_{S},(1, l)_{S}\right]=1
$$

for all $l \in K$. Hence,

$$
\left[\left(1, k^{\alpha^{-1}}\right)_{R},\left(1, z^{\alpha^{-1}}\right)_{R}\right]=1 \text { for all } z \in K \text {. }
$$

This implies $\left(1, k^{\alpha^{-1}}\right) \in Z(R)=z_{R}$, and we obtain $k^{\alpha^{-1}}=1$. Thus we have $k=1$, and $z(S)=z_{S}$. This finishes the proof.

\section{The group of autoclinisms}

Let $G$ be a group. The isoclinisms from $G$ to $G$ are called autoclinisms, and it is easy to see that the autoclinisms constitute a group, and that an isoclinism from $G$ to another group induces an isomorphism of the corresponding groups of autoclinism Thus, the group of autoclinisms is an invariant of the families of isoclinic groups. Now we can prove the following result of Hall, (cf. [3]). 
THEOREM 4.1. Let acl $(\Gamma)$ be the group of autoclinisms of tine family $\Gamma$. Let $S_{1}, S_{2}, \ldots, S_{n}$ be a complete system of nonisomorphic stem groups in $\Gamma$. Then we have

$$
\frac{I}{|\operatorname{acI}(\Gamma)|}=\sum_{i=1}^{n} \frac{1}{\left|\operatorname{aut}\left(s_{i}\right)\right|} \text {. }
$$

Proof. Let $R=(Z, K, r)$, where $Z_{R}=Z(R)$, be a stem group in $\Gamma$, and let $(\alpha, \beta) \in \operatorname{aut}(K) \times \operatorname{aut}(Z)$. By Theorem 3.2, $(\alpha, \beta)$ is induced by an autoclinism of $R$, if and only if there exists an $f \in \operatorname{sym}(K, Z)$, such that $r^{(\alpha, \beta)}$ and $r f$ are equivalent. Let $A$ be the subgroup of all $(\alpha, \beta)$ which satisfy the condition above. Then we have

$$
A \simeq \operatorname{acl}(\Gamma) \text {. }
$$

Let $(\alpha, \beta) \in A$ such that $r^{(\alpha, \beta)}$ and $r f$ are equivalent, and let $g \in \operatorname{sym}(K, Z)$. Then $(r g)^{(\alpha, \beta)}$ and $r(f g)^{(\alpha, \beta)}$ are equivalent and $(f g)^{(\alpha, \beta)} \in \operatorname{sym}(K, Z)$. Furthermore, if $s$ and $t$ are arbitrary factor sets which are equivalent, then $s^{(\alpha, \beta)}$ and $t^{(\alpha, \beta)}$ are equivalent.

For all factor sets $s$, let $\bar{s}$ denote the corresponding elements in $H^{2}(K, Z)$, and let

$$
\Omega=\bar{r} \cdot \overline{\operatorname{sym}}(K, Z)=\{\bar{r} \bar{f} \mid f \in \operatorname{sym}(K, Z)\} .
$$

The considerations above yield that

$$
\bar{s}^{(\alpha, \beta)}:=\overline{s^{(\alpha, \beta)}} \text {, for all } \bar{s} \in \Omega, \quad(\alpha, \beta) \in A \text {, }
$$

defines a permutation representation of $A$ on the set $\Omega$. Now we are going to study the orbits of $A$ and the point stabilizers.

Let $\bar{s}, \bar{t} \in \Omega$. By Theorem 3.2 we obtain that $S=(2, K, s)$ and $T=(Z, K, t)$ are stem groups in $\Gamma$. Let $(\alpha, \beta) \in A ;$ then $\bar{s}^{-(\alpha, \beta)}=\bar{t}$ implies by Lemma 1.3 that $S$ and $T$ are isomorphic. On the other hand, if $\lambda$ is an isomorphism from $S$ to $T$, we have $z_{S}^{\lambda}=z_{T}$, and $\lambda$ induces $\cdot(\psi, n) \in \operatorname{aut}(K) \times \operatorname{aut}(Z)$ such that $s^{(\psi, n)}$ and $t$ are equivalent. Let $f_{1}, f_{2} \in \operatorname{sym}(K, Z)$ such that $r$ is equivalent to $s f_{1}$ 
and $t$ is equivalent to $r f_{2}$. Then it is easy to see that $r^{(\psi, n)}$ and $r f_{1}^{(\psi, n)} f_{2}$ are equivalent, and $f_{1}^{(\psi, n)} f_{2} \in \operatorname{sym}(K, Z)$. This implies $(\psi, \eta) \in A$. Hence the orbits of $A$ correspond to the isomorphism classes of stem groups in $\Gamma$.

Let $\Omega_{1}, \Omega_{2}, \ldots, \Omega_{n}$ be the orbits of $A$, and let $\bar{s}_{i} \in \Omega_{i}$ and $s_{i}=\left(z, k, s_{i}\right)$. Then $S_{1}, S_{2}, \ldots, S_{n}$ is a complete system of stem groups of $\Gamma$. Let $(\alpha, \beta) \in A$, and assume $\vec{s}_{i}^{(\alpha, \beta)}=\bar{s}_{i}$. By Lemma 1.3 , this relation holds if and only if $(\alpha, \beta)$ is induced by an automorphism of $S_{i}$. As each autoclinism of a group is determined by the operation on the central factor group, two automorphisms of the group induce the same autoclinism, if and only if they differ only by a central automorphism. Hence we obtain for the stabilizer of $\bar{s}_{i}$ :

$$
\operatorname{stab}\left(s_{i}\right) \simeq \operatorname{aut}\left(s_{i}\right) / C\left(S_{i}\right)
$$

Thus we have

$$
|\Omega|=\sum_{i=1}^{n}\left|\Omega_{i}\right|=\sum_{i=1}^{n}\left|A: \operatorname{stab}\left(\bar{s}_{i}\right)\right|=\sum_{i=1}^{n} \frac{|A| \cdot\left|c\left(S_{i}\right)\right|}{\left|\operatorname{aut}\left(s_{i}\right)\right|} .
$$

By (2.2) we have for all $i$,

$$
\left|C\left(S_{i}\right)\right|=|\overline{\operatorname{sym}}(K, Z)|=|\Omega| \text {. }
$$

This finishes the proof.

\section{References}

[1] L. Fuchs, Abelian groups, 3rd ed. (Pergamon Press, Oxford, London, Edinburgh, New York, Toronto, Sydney, Paris, Braunschweig, 1960; reprinted 1967).

[2] P. Hall, "The classification of prime power groups", $J$. reine angew. Math. 182 (1940), 130-141.

[3] P. Hall, "On groups of automorphisms", J. reine ongew. Math. 182 (1940), 194-204. 
[4] B. Huppert, Endliche Gruppen I (Die Grundlehren der mathematischen Wissenschaften, 134. Springer-Verlag, Berlin, Heidelberg, New York, 1967).

[5] Ra†je Reimers, "Die Berechnung der Stammruppen einer Isoklinismenfamilie", (Diplomarbeit, Aachen, 1972).

[6] P.R. Sanders, "The central automorphisms of a finite group", J. London Math. Soc. 44 (1969), 225-228.

Institut für Reine und Angewandte Mathematik, Rheinisch-Westfäliscne Technische Hochschule, Aachen,

Germany;

Lehrstuhl D für Mathematik,

Rheinisch-Westfäl ische Technische Hochschule, Aachen,

Germany. 